\title{
Variability of the Light Curves of the Close Binary GR Tauri
}

\author{
A. Yamasaki, M. Takeda, T. Yamauchi, G. Takada, S. Hattori \\ Department of Earth and Ocean Sciences, National Defense Academy, \\ Yokosuka 239-8686, Japan
}

\begin{abstract}
Variability of the light curves of the short-period eclipsing binary system GR Tau ( $P=0 \mathrm{~d} 43, \mathrm{~A} 5 / \mathrm{A} 9$, almost-contact binary) is studied. It is found that GR Tau experienced both the state which is characterized by asymmetric light curves and the state characterized by symmetrical light curves.
\end{abstract}

\section{Photometry of GR Tau}

GR Tau (BD $+20^{\circ} 685=$ TYC 1258-00505-1, $\left.V=10.26, P=0.43\right)$ is an eclipsing binary of EB/SD: type. From the viewpoint of the binary evolution off the main sequence, a study of GR Tau is important because of its early spectral type and short period. Yamasaki et al. (1984) obtained light curves with a photometric asymmetry and classified the spectral type as A5 while Lázaro et al. (1995) obtained symmetrical light curves and the later spectral type A9 from the color. Photometry of GR Tau was also made by other workers (Hanžl 1990; Fang et al. 1994) at different epochs against the same comparison star $\left(\mathrm{BD}+20^{\circ} 684\right)$. Among them both light curves of Yamasaki et al. and Lázaro et al. are quite dissimilar to each other, so we continued photometric monitoring of this interesting star. Our observations made at Yokosuka using a CCD are summarized in Table 1.

Table 1. CCD Observations at Yokosuka

\begin{tabular}{llll}
\hline Period & Observers & Telescope & Filters \\
\hline 1995 Dec-1996 Jan & Yamauchi \& Yamasaki & $0.3 \mathrm{~m}$ & $B V$ \\
1996 Oct-1997 Feb & Takada, Hattori, \& Yamasaki & $0.3 \mathrm{~m}$ & $B V$ \\
1999 Oct-2000 Jan & Takeda \& Yamasaki & $0.2 \mathrm{~m}$ & $B V R$ \\
\hline
\end{tabular}

\section{Light Curves Observed at Different Epochs}

Magnitudes at MaxI (0.25), MaxII (0.75), MinI (0p0), MinII (0.5) and depths of both minima are found to vary with time. Lázaro et al.'s (and Hanžl's) light curves are symmetrical and depths of minima are shallower while other light curves show asymmetry and deeper depths of minima. Light curves of GR Tau observed so far may be classified into two types, asymmetrical (A) 
and symmetrical (S). Each type of the light curve has the features described in Table 2. A detailed report on the subject will be published elsewhere.

Table 2. Light Curve Types

\begin{tabular}{lll}
\hline & Asymmetrical light curve (A) & Symmetrical light curve (S) \\
Maxima & MaxII fainter & Equal \\
Minima & Deeper, total & Shallower, partial \\
Color & Bluer & Redder \\
Obs. & 1980 Yamasaki et al. & 1989 Hanžl \\
& 1993 Fang et al. & 1992 Lázaro et al. \\
& $1995 / 1996$ Yamauchi \& Yamasaki & \\
& $1996 / 1997$ Takada et al. & \\
\hline
\end{tabular}

\section{References}

Fang, M., Zhai, D., Gao, Z., Zhang, R., Zhang, J., \& Zhang, X. 1994, IBVS, 4082

Hanžl, D. 1990, IBVS, 3423

Lázaro, C., Niarchos, P., Rovithis, P., Rovithis-Livaniou, E., Arévalo, M. J., \& Antonopoulou, E. 1995, AJ, 110, 1796

Yamasaki, A., Okazaki, A., \& Kitamura, M. 1984, PASJ, 36, 175 